Involvement (PPI), this project aimed to understand parents' opinions and experiences around their child's sleep and sleep interventions in order to tailor a BSI for use in the CASTLE (Changing Agendas on Sleep, Treatment and Learning in Epilepsy) study clinical trial with CWE.

Method Semi-structured interviews were conducted with 10 mothers of CWE and $3 \mathrm{CWE}$ recruited via online adverts on epilepsy relevant websites. A thematic analysis was conducted on the interview data.

Results Several strong themes were apparent including that i) families felt in need of information, support and help with sleep, ii) parents valued other parents' experience/understanding (over professionals' advice), iii) any intervention needed to be personalised and non-prescriptive, and iv) parents' concerns needed to be included. Two theory-driven BSIs, used in evidence-based level 1 studies, were adapted to incorporate parents' requirements, developed into an online intervention (CASTLE Online Sleep Intervention or COSI) for parents of CWE and refined following parental evaluation (via online questionnaire built into COSI) until $100 \%$ approval from parents was reached.

Discussion Adaptations and additions to content and delivery format were necessary to ensure that COSI best met parents' needs; the final version will be used in the CASTLE study clinical trial (http://castlestudy.org.uk/). It is hoped that the use of evidence-based techniques delivered with consideration of factors identified through PPI, can increase parent-engagement and optimise effectiveness.

\section{P012 A FEASIBILITY STUDY OF THE USE OF AN EXERCISE INTERVENTION TO IMPROVE WELL BEING USING A MOBILE APP FOR MONITORING PATIENT RELATED OUTCOMES IN CHILDREN WITH NARCOLEPSY}

${ }^{1}$ Rosalind Broe*, ${ }^{2}$ Janine Reynolds, ${ }^{2}$ Heather Elphick. ${ }^{1}$ The Medical School, University of Sheffield, Sheffield, UK; ${ }^{2}$ Sheffield Children's NHS Foundation Trust, Sheffield, UK

10.1136/bmjresp-2019-bssconf.12

Introduction Narcolepsy is a chronic neurological condition in which the brain's ability to regulate the sleep-wake cycle is disturbed and can result in reduced quality of life (QOL). A positive association between exercise and QOL has been found in children. The aim of this study was to assess the feasibility of implementation of an exercise intervention with a mobile app to monitor outcomes in children with narcolepsy.

Methods This feasibility study received service evaluation (SE1308), and University of Sheffield ethical approval (project number: 022995). A patient and parent engagement exercise to inform study design was carried out during the 'Sheffield Children's Hospital Annual Narcolepsy Family Day'. An exercise intervention was designed based upon NHS recommendations and tailored to the child's activity levels. ${ }^{1}$ Weekly goals were set and progress was reviewed weekly, using an app specifically developed by Aparito Ltd (Wrexham, UK). QOL was monitored using the Pediatric Quality of Life Inventory (PedsQL). Patients were asked for feedback at the end of the intervention, to assess its feasibility and acceptability.

Results 117 children and parents took part in the engagement exercise. 12 patients took part in the feasibility study (mean age 12.9 years). The focus group highlighted the importance of motivation, timing and tailoring exercise interventions to the individual. Children said exercise should be fun to help motivation.

Average physical activity increased from 229 minutes to 254 minutes. Of those who had successfully increased their exercise, baseline average PedsQL score increased from 70.6 to 77.6 (figure 1). Feedback on the app and exercise intervention were both positive.

Conclusion This feasibility study has shown that a targeted exercise intervention can improve QOL in children with narcolepsy. The Aparito app, it is being developed further for children with narcolepsy.

\section{REFERENCE}

1. Health Survey for England 2015: Physical Activity for Children

\section{P013 A NURSE-LED BEHAVIOURAL SLEEP PROGRAMME CAN REDUCE MELATONIN PRESCRIBING}

Aditi Sivaramakrishnan, Janine Reynolds*, Rosalind Broe, Ruth N Kingshott, Heather E Elphick. Sheffield Children's NHS Foundation Trust, Western Bank, Sheffield, UK

\subsection{6/bmjresp-2019-bssconf.13}

Introduction Many children with sleep difficulties are prescribed melatonin. However, melatonin is costly and evidence for its efficacy is limited. The aim of this study was to

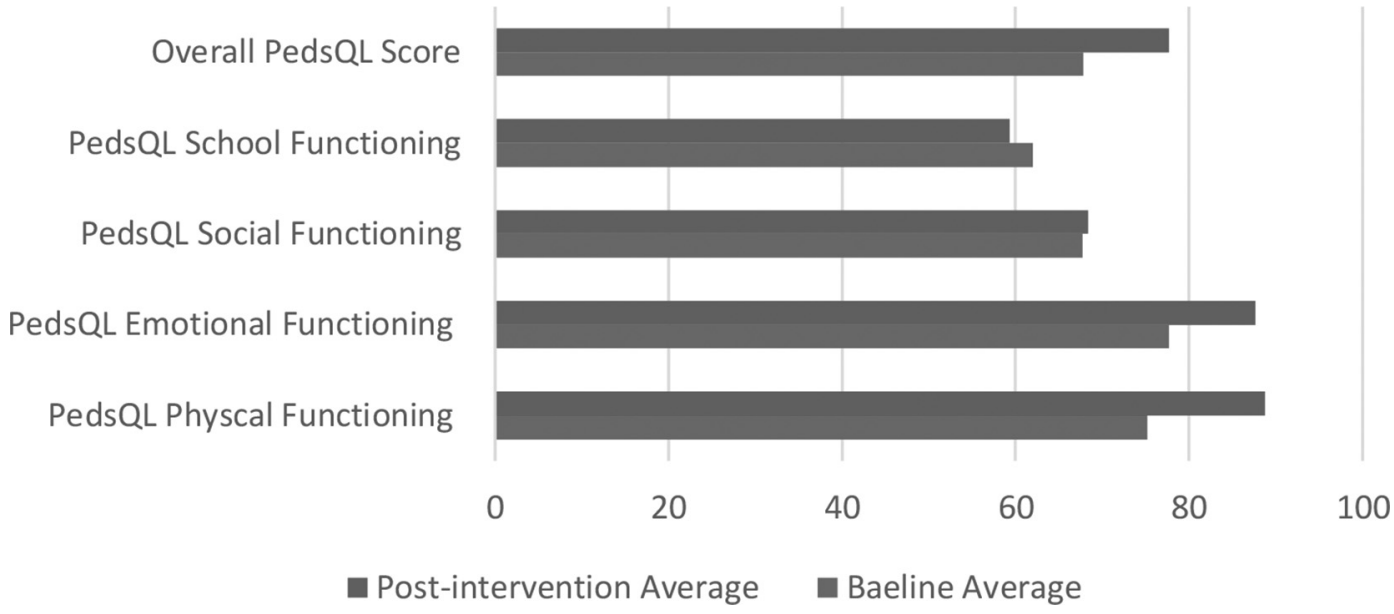

Abstract P012 Figure 1 Average Baseline and Post-intervention PedsQL Scores (Successful Interventions) 